\title{
INDETERMINACY AND VAGUENESS: LOGIC AND METAPHYSICS
}

\author{
PETER VAN INWAGEN
}

\author{
University of Notre Dame
}

Vagueness is a special case of indeterminacy — semantical indeterminacy. It may be indeterminate whether a sentence is true or false, indeterminate whether a term denotes a certain object, and indeterminate whether a given set is the extension of a certain predicate. I take the word 'vague'my universe of discourse here comprises only linguistic items- to be entirely appropriate only in application to predicates and certain of their constituents. ${ }^{1} \mathrm{~A}$ predicate is vague if it is indeterminate, or, at any rate, possibly indeterminate, which set is its extension - or if it is possible that, for at least one object, it is indeterminate whether that object belongs to the extension of that predicate. In other words, a predicate is vague if it admits of (potential) borderline cases. In the case of one-place predicates, this comes down to saying that the term 'vague' applies primarily to verbs and adjectives and prepositions, the main constituents of predicates. (Of course, if it applies to verbs and adjectives, it applies to adverbs as wellthat is, to representatives of the grammatical categories "takes a verb and makes a verb" and "takes an adjective and makes an adjective.")

\footnotetext{
${ }^{1}$ I concede that we speak of statements as vague- but when we say that a statement is vague, we mean that it is insufficiently explicit about some matter, that it lacks relevant specifics that a statement on the topic in question might have been expected to include. For example, one might well complain that a statement made by a public official— "There appear to have been certain irregularities in the Minister's conduct of his office,"let's saywas vague. But one would not be taken to mean that the statement was of indeterminate or indefinite truth-value: no doubt the speaker would be willing to grant that it was definitely true that there appeared to have been certain irregularities in the Minister's conduct of his office. And, while "appearance of irregularity in the conduct of a ministerial office" certainly admits of borderline cases, this fact would not be the fact that the person who complained about the vagueness of the statement about the appearance of irregularity in the conduct of a ministerial office was calling attention to.
} 
I will restrict the application of the term 'vague' to items that belong to the grammatical categories I've roughly delineated-predicates, verbs, adjectives, prepositions, and adverbs. I deprecate, in particular, any attempt to describe sentences as "vague," and I deprecate sentence operators like 'it is vague whether' (or, worse, 'it is vague that'). I am, however, happy to concede that my distaste for such usages is more a matter of my respect for the niceties of traditional English usage than a matter of logic or philosophy. I might mention in this connection-I need to mention it somewhere, and this seems as good a place as any-David Lewis's statement that the truth-functional connectives and the "idioms of quantification" are not vague. ${ }^{2}$ As I see matters, this is a sort of category mistake. Since neither the connectives nor the quantifiers have semantical values of any sort, I don't see what can be meant by saying either that they're vague or that they're not-or by saying that they do or that they don't exhibit indeterminacy. Possibly all that Lewis meant by saying that the connectives were not vague is that if a truth-functionally compound sentence is of indeterminate truth-value, this can only be because one or more of its truth-functionally simple constituents is of indeterminate truth-value. And that would certainly not be a category mistake. And, possibly, by saying that the idioms of quantification were not vague, he meant only that if one examines a sentence that starts with, say, an existential quantifier-phrase whose scope is the remainder of the sentence, and if one is convinced that that sentence is of indeterminate truth-value, one will have to say that it's of indeterminate truth-value because-and only because-it's indeterminate whether anything satisfies the open sentence whose variable the quantifier-phrase binds. I'll presently deny that thesis, but I certainly don't want to say that it exhibits any sort of category mistake.

Perhaps I should also say this: in restricting my application of the terms 'indeterminacy' and 'vagueness' to linguistic items, I don't mean to imply that these terms cannot be usefully applied to, say, attributes or relations or Fregean concepts and other non-linguistic abstract objectsparticularly those that belong to categories that (like the three categories I've mentioned) are intimately connected with predicates.

\footnotetext{
${ }^{2}$ On the Plurality of Worlds (Oxford: Blackwell, I986), p. 2 I2.
} 
To return to the topic of the vocabulary I recommend for discussions of indeterminacy, I would contend that the best sentence operator to employ when making an assertion to the effect that something or other is indeterminate is 'it is indeterminate whether' - or, at any rate, something very much like it. I suppose, however, that it is better for the primitive operator in any area of semantics or logic to have a positive rather than a negative form, so let the primitive operator be 'it is determinate whether'-despite the fact that 'it is indeterminate whether' seems a much more natural form of words than 'it is determinate whether'. It is determinate whether $p$ just in the case that the proposition that $p$ is definitely or determinately true or definitely or determinately false-or, if you like, if the question whether $p$ can be answered Yes (without qualification) or No (without qualification).

Determinacy and indeterminacy obviously have a logic, and it is usual to use the methods of formal semantics to describe the features of a logic. Formal semantics is the discipline whose task is to assign semantical values to sentences on the basis of the semantical values assigned to their syntactical components. ${ }^{3}$ When the operator 'it is determinate whether' ('DET') is applied only to closed sentences, its semantics is simple, and, I hope, uncontroversial. It can be presented in a simple value-table:

$\begin{array}{lccc}p & \sim p & \text { DET } p & \text { INDET } p[=\sim \text { DET } p] \\ \circ & \mathrm{I} & \mathrm{I} & \mathrm{O} \\ 1 / 2 & 1 / 2 & \mathrm{O} & \mathrm{I} \\ \mathrm{I} & \mathrm{O} & \mathrm{I} & \mathrm{O}\end{array}$

In this table, 'I' represents determinate truth or truth without qualification, 'o' represents determinate falsity or falsity without qualification, and ' $1 / 2$ ' represents the condition "being neither determinately true nor determinately false."

${ }^{3}$ More exactly, that task is the "core" or central task of formal semantics. Once one has decided how to assign semantical values to sentences on the basis of the semantical values of their components, one may go on to assign semantical values to sequences of sentences (to arguments or inferences) on the basis of the semantical values of the syntactical components of the members of the sequences-values like 'valid' or 'invalid', for example. 
It is certainly true - determinately true - that we make assertions that are neither determinately true nor determinately false. No one would deny that in many cases, no doubt in most cases, the fact that an assertion is of indeterminate truth-value has its ground in language. But is this always the case? I will try to answer this question. The first step of my attempt to answer it will be an outline of what I what I will call the "sensible" theory of indeterminacy.

The sensible theory of indeterminacy (sc. of truth-value) is that indeterminacy of truth-value is entirely a matter of the semantical values of the syntactic constituents of sentences being underdetermined by the conventions that govern the assignment of those values. Consider, for example, predicates. (From this point to the point at which I explicitly resume speaking in propria persona, I will speak in the voice of an adherent of the sensible theory.) To specify the meaning of a predicate is to give a set of instructions for its application, and it is well-nigh impossible for a set of instructions to cover every possible situation; in consequence, no matter how carefully we specify the rules for using some new predicate that we propose to introduce into our language, there will almost certainly be possible cases in which it is indeterminate whether that predicate applies. (And, as many writers have pointed out, when one introduces a new predicate, there will normally be good, practical reasons for leaving it indeterminate whether it applies in possible cases in which one could render its application determinate. As Lewis has said, no one has ever been fool enough to try to specify a precise portion of the surface of the earth as the referent of 'the outback'. ${ }^{4}$ ) It would seem, therefore, that all or almost all predicates will admit of possible borderline cases; and many predicates will have actual borderline cases. It is these actual borderline cases that account for all actual cases of indeterminacy-that is, all cases of assertions that are syntactically and semantically unobjectionable and are yet neither determinately true nor determinately false. (Someone's statement that Fred is bald, say, or that Mary is tall.)

I have said that "all or almost all" predicates will admit of possible borderline cases. Might all predicates have possible borderline cases? Pure mathematics provides a class of possible counterexamples to the

${ }^{4}$ Loc. cit. 
thesis that all predicates have possible borderline cases, as do theology and Platonic metaphysics and theoretical physics ('electron', 'neutrino'). I will not discuss cases of that sort. Those cases aside, there are certain special predicates that have and can have no borderline cases. These are the predicates that can be constructed using only the language of firstorder logic - that is, first-order logic with identity, for it is only when the identity-sign has been added to the language of logic that it is possible to construct predicates entirely out of logical materials. Two important examples are ' $x=x$ ' and ' $x=y$ '. (I'm not going to bother to distinguish between predicates and the open sentences that are their typical instances.) The former expresses the attribute of existence (being equivalent to ' $\exists y y=x$ ', at least given the usual formulation of the rule of existential generalization), and the latter the relation of identity. These predicates have no borderline cases, for existence and identity have no borderline cases. "Identity, properly speaking, knows no gradation," says Quine, ${ }^{5}$ and Chisholm has said more or less the same thing about existence. ${ }^{6}$ It is predicates whose meaning is specified by a set of instructions (instructions that determine - insofar as anything determines this - whether that predicate applies to a given object or sequence of objects) that are vague, that have possible or actual, borderline cases. There can be no borderline cases of existence, because an object has to be there to be a borderline case of anything, and if it's there it exists. There can be no borderline cases of identity because an object $x$ and an object $y$ are either two objects or one; if they are two, they are not identical, and if they are one they are. If there were borderline cases of existence, there would be sets each of which was such that it was indeterminate whether it was the empty set or a unit set. If there were borderline cases of identity, there would be sets each of which was such that it was indeterminate whether it had one or two members. And these things are simply impossible. All indeterminacy is a product of vagueness (the vagueness that comes from vaguely drawn boundaries), and vagueness takes up only where logic has left off - and, therefore, indeterminacy takes up only where logic has left

\footnotetext{
${ }^{5}$ Word and Object (Cambridge, Mass.: The M.I.T. Press, I960), p. 203.

${ }^{6}$ See his essay, "Coming into Being and Passing Away" in On Metaphysics (Minneapolis: University of Minnesota Press, I989), especially the section entitled "Elanguescence," pp. $55^{-56}$
} 
off. Vagueness arises when we draw boundaries and arises because it is humanly impossible to draw any boundary such that every possible object falls either definitely inside or definitely outside that boundary. But in logic there is no drawing of boundaries.

Here endeth the statement of the sensible theory of indeterminacy. I resume speaking in propria persona.

In my view, the sensible theory of indeterminacy, appealing as it is, cannot accommodate a workable metaphysic of the material world. Any attempt to spell out in detail a metaphysic of the material world that incorporates the sensible theory of indeterminacy (which denies that there can be indeterminate cases of identity and existence) will demonstrably have consequences less appealing, or more appalling, than a rejection of the sensible theory of indeterminacy. There is a lot that could be said about this. I could write a book. Here I must content myself with an example. When we attempt to construct a metaphysic of the material world, one of the questions we must answer is the Special Composition Question: "When are things proper parts-when do things together compose some larger whole?" Suppose, just for the sake of having an illustration, that we say that things compose a larger whole when and only when they are in physical contact. (Thus, twenty blocks spread about on a floor compose nothing; when a child builds a tower out of them, they compose something: a tower of blocks.) Now suppose the world consists of two cubical blocks - each of exactly the same dimensions as the other-floating about in otherwise empty space; and suppose that at one time they are not in contact and that a moment later they drift together and are in contact. If current physics is correct, there must have been some moment $t$ at which it was indeterminate whether they were in contact. (By "current physics," I do not mean quantum mechanics, or at least I am not thinking primarily of quantum mechanics-I am referring to facts about the structure of matter that were known well before the advent of quantum mechanics.) Now consider the moment $t$ - a moment at which it is indeterminate whether the two blocks are in contact. Ask this question: Does anything larger than either of the two blocks exist at $t$ ? It cannot be definitely true that there then exists something larger than either block, for that could be the case only if there were definitely something the two blocks were parts of; and there 
could definitely be something the two blocks were parts of only if the two blocks were definitely in contact. A parallel argument shows that it cannot be definitely false that there then exists something larger than either block. So we have a case of indeterminacy - from the point of view of our simple possible world, an actual case. According to the sensible theory of indeterminacy, this must be because there is, in our miniature world, something that is a borderline case of "is larger than either block." But what is it? It is not either of the blocks, each of which is a determinate case of "is not larger than either block." And if the two blocks have proper parts, it certainly isn't any of them. Could it be the fusion or mereological sum of the blocks, the thing they compose, the thing that has them both as parts and each of whose parts overlaps at least one of them? This suggestion will not do because it is not at $t$ determinately true that there is such a thing, and we are thus not in a position to assert, "The sum of the blocks is at $t$ a borderline case of 'is larger than either block'." (And, of course, even if we were in a position to make assertions implying the existence at $t$ of the sum of the blocks, this would not enable us to explain the case of indeterminacy we want to explain, for the sum of the blocks would not be a borderline case of 'is larger than either block'; it would be quite definitely twice the size of either of the blocks.) Our little possible world seems to contain no other candidate for the office "is a borderline-case of "is larger than either block'." It seems, indeed, to contain no even remotely plausible candidate for that office. It would appear, therefore, (a) that the assertion "There exists something larger than either block" is of indeterminate truth-value, and (b) that we cannot explain this indeterminacy by saying "There is something that is a borderline case of 'is larger than either block'."

It is instructive to compare this example with a case of indeterminacy in which the sensible theory seems to provide a correct explanation of that indeterminacy. Suppose that Socrates is "borderline wise," and that no one is determinately wise. Then it is indeterminate whether there is anyone who is wise, and the explanation is a straightforward one: there exists someone-Socrates-such that it is indeterminate whether the predicate 'is wise' applies to that person, and there exists no one such that the predicate 'is wise' determinately applies to that person. But in the "two blocks" case, I cannot make the assertion that corresponds to 
"There exists someone such that it is indeterminate whether the predicate "is wise' applies to that person": I cannot say, "There exists something such that it is indeterminate whether the predicate 'is larger than either block' applies to that thing."

If the sensible theory is correct, however, the only way to explain the indeterminacy of truth-value of 'There exists something larger than either block' is to assert the existence of an object such that it is indeterminate whether 'is larger than either block' applies to it. If our simple possible world is indeed possible, therefore, the sensible theory is wrong. In our simple possible world, existence is indeterminate: it is indeterminate whether there exists a mereological sum of the two blocks, and not because there exists something that is a borderline case of 'is a mereological sum of the two blocks'. And the idea of indeterminate existence is a mystery; we understand indeterminacy, at least to some degree, when it can be explained by reference to vaguely drawn boundaries; but cases of indeterminate existence cannot be explained by reference to vaguely drawn boundaries.

So: there are sentences in which one variable is free that have both the following properties:

- The existential generalization on those sentences is of indeterminate truth-value.

- Their existential generalizations' being of indeterminate truth-value cannot be explained by an appeal to objects that "borderline satisfy" them.

It is in that sense that existence is indeterminate-there are such open sentences. (Or there are at least sentences that, in certain possible circumstances, would have those properties.) When I contend that existence can be indeterminate, I mean only that much. I do not mean that there are or could be objects that are or would be borderline cases of existence. There cannot be an object that borderline-satisfies ' $\exists y y=x$ '. (At any rate, there cannot definitely or determinately be an object that borderline-satisfies this sentence-and, therefore, anyone who agrees with very much of what I have said will not be in a position to use the sentence "There is an object that borderline-satisfies " $\exists y y=x$ ” to make an assertion. One 
might argue about whether this metalinguistic or semantical sentence might, if circumstances cooperated, be of indeterminate truth-value, but there are certainly no circumstances in which it would be determinately true. $^{7}$ ) There are, therefore, sentences whose philosophical import can be summed up in the slogan "Existence can be indeterminate." There is, however, no reason to say that existence can be vague (or to use phrases like 'the vagueness of existence' or 'vague existence')- for the slogan "existence can be vague" strongly suggests that the sloganeer thinks that there are possible circumstances in which there would be borderline existents.

Since the case of indeterminacy - existential indeterminacy-we have considered cannot be grounded in language, it seems fair to describe it as a case of ontic indeterminacy.

I contend this: any carefully worked-out metaphysic of the material world will either present us with cases of existential indeterminacy or else will have consequences that embody even more unpalatable mysteries than the mysteries that attend existential indeterminacy. (For example, it may imply that there are no such things as you or I, or that for every material thing $x$, it is a necessary truth that for every moment $t$, it is either determinately true or determinately false that $x$ exists at $t$.) Rather than accept any of these consequences and confront the mysteries that follow in their wake, I prefer to accept the reality of existential indeterminacy. If existential indeterminacy is a phenomenon that is not well understood (to borrow a euphemism from the sciences), it is certainly not the only one. After all, no one really understands such staples of philosophical discourse as self-reference, consciousness, time, and free will. If we do not understand something, the thing to do is to own up to that fact, and not to insist that that "something" does not exist.

If there are sentences whose philosophical import can be epitomized in the slogan, "Existence can be indeterminate," there are also sentences whose philosophical import can be epitomized in the following two slogans: "Identity can be indeterminate"; "Identity can be vague."

\footnotetext{
${ }^{7}$ Return to the case of the two blocks. Consider the moment $t$ at which the two blocks are in "borderline contact." It is plausible to suppose that the sentence 'There is an object that borderline-satisfies " $\exists y y=x$ " is of indeterminate truth-value at $t$. However that may be, there is certainly never a time at which that semantical assertion is determinately true.
} 
An example of Terence Parsons's shows that there are such sentences:

Suppose I am driving down the freeway, and suddenly swerve to avoid a pile of trash. The cleanup crews show up later, and push around a lot of stuff-some of which made up the pile I swerved around, as well as some other stuff. The next day I drive by a pile of trash. Is it the same pile as the pile that was there yesterday? In some cases of this sort, the question has no apparent answer. ${ }^{8}$

We can recast Parsons's metaphysical question as a semantical question. Suppose that, having passed the pile of trash on "the next day," Parsons utters the following sentence: "The pile of trash I swerved to avoid yesterday = the pile of trash I drove by today."

Is this sentence ("the Parsons sentence") true or false? Like the metaphysical question, this semantical question may well have no apparent answer. Let us suppose that it does not. If it does not, that is because it has no determinate answer. (The question, 'Is the number of ' 7 's in the first trillion digits of the decimal expansion of $\pi$ odd or even?' has no apparent answer-no answer that is apparent to any reader of this paper, at any rate-, but it has a determinate answer.) There are, therefore, identity sentences of indeterminate truth-value. ${ }^{9}$

Can the indeterminacy of sentences like the Parsons sentence be accounted for by the sensible theory of indeterminacy? Well, it can if one is willing to adopt a perdurantist account of identity across time. But that account involves its adherents in various mysteries (for example, that each of us has a certain precise span of existence-like 8I years, I4

8 "Entities without Identity," Philosophical Perspectives r: Metaphysics (1987), pp. I-I9. See p. 3 .

${ }^{9}$ Since I myself don't - in the ontology room-believe in piles of trash, since I believe that, speaking strictly and ontologically, every pile of trash is definitely non-identical with every pile of trash, in my own discussion of indeterminate identity I imagined an example involving an indeterminate number of human beings and an infernal device called the Cabinet. But the logical point Parsons's example was intended to make and the logical point my example was intended to make are the same. My discussion of the possibility of indeterminate identity can be found in Material Beings (Ithaca: Cornell University Press, I990), Section I8, pp. 228-270. 
days, II hours, 53 minutes, and eight and one half seconds-essentially ${ }^{10}$ ). I will not discuss that option. I will simply suppose that, for one reason or another, one is not willing to adopt perdurantism; in that case, might one account for the indeterminacy of the Parsons sentence in terms acceptable to proponents of the sensible theory of indeterminacy? It might seem so. For, if one is persuaded by Parsons's case, one will come to the conclusion that the indeterminacy of the sentence can be traced to the vagueness of a predicate that occurs in that sentence, to wit, the identity predicate. After all, if one is persuaded to see things as Parsons sees them, one will be persuaded that identity has borderline cases: the objects the pile of trash I swerved to avoid on Tuesday and the pile of trash I swerved to avoid on Friday constitute a borderline case of two objects and therefore, taken together, taken as a pair ${ }^{11}$, constitute a borderline case of identity. But, as we have seen, it is an essential component of the sensible theory of indeterminacy that the predicate ' $x=y$ ' cannot admit of borderline cases.

If the Parsons sentence is indeed of indeterminate truth-value (we are assuming, remember, that its right-hand term and its left-hand term both definitely denote something), it presents us with a case of ontic indeterminacy, indeterminacy whose ground is in the world and not in language. Existential indeterminacy and indeterminacy of identity are two kinds of ontic indeterminacy (the only two of which I am aware).

If the Parsons "piles of trash" case is a case of ontic indeterminacy, it is also a case of ontic vagueness-for if the Parsons sentence is of indeterminate truth-value (and if its right-hand term and its left-hand term

${ }^{10}$ See my essay "Four-dimensional Objects," Nô̂s 24 (1990), pp. 245-255. The consequence mentioned in the text can be avoided if one adopts an anti-realist account of modality de re (such as counterpart theory with multiple counterpart relations). But the idea that there is no fact of the matter as to what a thing's essential properties are is a very mysterious idea-much more mysterious than the idea of indeterminate identity. Or so say I.

11 "Taken as a pair" is, I concede, loose talk. A pair, I suppose, is a two-membered set. But either there is no such set as \{the pile of trash Parsons swerved to avoid yesterday, the pile of trash Parsons drove by today\}, or, if there is such a set, it is indeterminate whether it has one member or two. In either case, it is far from evident what it could mean to speak of taking the pile of trash Parsons swerved to avoid yesterday and the pile of trash Parsons drove by today "as a pair." 
both definitely denote something), then the identity predicate is vague. Although the existence predicate ( $x=x^{\prime}$ ' or ' $\exists y y=x^{\prime}$ ) does not admit of borderline cases, the identity predicate (' $\left.x=y^{\prime}\right)$ does.

It is true, of course, that the sensible theory of indeterminacy can account for some indeterminate identity-sentences-just as it can account for some indeterminate existential sentences. (Some early critics of Gareth Evans's famous argument for the impossibility of vague identity apparently thought that he was trying to prove the obviously false thesis that there could not be any identity-sentences of indeterminate truthvalue. $\left.{ }^{12}\right)$ Suppose, for example that in 1792 it was indeterminate whether Louis XVI (or "Citizen Capêt") reigned over France, and determinately true that no other person did. If, then, someone had said in I792, "The present King of France $=$ the King of France in 1782 ," what that person said would have been of indeterminate truth-value. And, of course, the sensible theorists have no trouble accounting for that: there is a certain object such that, in 1792 , at the moment the sentence was uttered, it was determinately true that 'the King of France in I 782 ' denoted that object and indeterminate whether 'the present King of France' denoted that object. But there is an important semantical difference between the "King of France" sentence and the Parsons sentence. It's indeterminate whether the phrase 'the present King of France' (uttered in 1792) denotes the King of France in 1782 (that is, denotes the man who held that royal office in 1782 ) because it's indeterminate whether it denotes anyone. (More exactly, because it has the following feature: There is some $x$-at least one-such that it's indeterminate whether it denotes $x$, and there's no $x$ such that it determinately denotes $x$.) It's indeterminate whether 'the pile of trash I passed by today' (uttered by Parsons on a certain day) denotes the pile of trash Parsons had swerved to avoid on the previous day. But this fact cannot be explained by saying that it's indeterminate whether 'the pile of trash I passed by today' denotes anything at all. For it quite definitely does denote something - the unique pile of trash that Parsons passed on the day he used that denoting phrase. The identity-sentences whose indeterminacy the sensible theory cannot account for are those

${ }^{12}$ See David Lewis, "Vague Identity: Evans Misunderstood," Analysis 48 (1988), pp. I28-I3O. 
each of whose terms definitely denotes something-sentences like the Parsons sentence or the sentence that figured in my "Cabinet" example. (In the latter case, one of the terms of the sentence definitely denotes the person who entered the Cabinet and the other definitely denotes the person who emerged from the Cabinet.)

I alluded a moment ago to Gareth Evans's clever argument for the impossibility of vague identity. ${ }^{13}$ You know: if Trashers is a pile of trash and Rubbers is a pile of trash, it can't be indeterminate whether Trashers and Rubbers are identical. For suppose it is indeterminate. Then Trashers has the property of being (only) indeterminately identical with Rubbers. But Rubbers obviously does not have the property of being (only) indeterminately identical with Rubbers. ${ }^{14}$ It therefore follows from the premise that Trashers and Rubbers are indeterminately identical that Trashers has a property Rubbers lacks and is therefore not identical with Rubbers. Now the proponents of indeterminate identity will want to assert the sentence 'It is indeterminate whether Trashers is identical with Rubbers'. And one should be willing to assert anything one recognizes as validly deducible from something that one is willing to assert. The friends of indeterminate identity, therefore, should be willing to assert that Trashers is not identical with Rubbers. But one should be willing to assert something only if one regards it as determinately true. The friends of indeterminate identity should, therefore, regard 'Trashers is not identical with Rubbers' as determinately true and 'Trashers is identical with Rubbers' as determinately false-and should, in consequence, regard 'It is indeterminate whether Trashers is identical with Rubbers' as determinately false. Therefore, anyone who accepts the thesis that Trashers is indeterminately identical with Rubbers is committed - if only pragmatically — to accepting the denial of that thesis. If the friends of indeterminate identity are indeed in this position, it is unlikely that they will regard themselves as in a state of philosophical equilibrium.

\footnotetext{
13 “Can There be Vague Objects?" Analysis 38 (1978), p. 208.

${ }^{14} \mathrm{Or}$, if you don't like the idea of properties that involve individuals, suppose that Rubbers contains an empty Blue Bull Bitter bottle and that Trashers does not. Then Trashers has the property of being (only) indeterminately identical with something that contains an empty Blue Bull Bitter bottle and Rubbers does not have that property.
} 
The question this argument has always brought to my mind is: Which of the following two theses is the more plausible - that cases like Parsons's "piles of trash" case fail to establish the real possibility of indeterminate identity or that there is an invalid step (at least one) in Evans's deduction of 'Trashers is not identical with Rubbers' from 'It is indeterminate whether Trashers is identical with Rubbers'? And it has always seemed to me that the answer is obvious - at any rate the prima facie answer. The prima facie answer, the default answer, the answer one should accept unless there should turn out to be something seriously wrong with it - demonstrably seriously wrong with it-is that the thesis that there's something wrong with Evans's argument is the more plausible of the two. Evans's argument, in my view, is in much the same position as Zeno's arguments: in each case, we may ask, "For what is a man profited if he shall present an a priori demonstration of the non-existence of $x$ and there's an $x$ right before the eyes of his audience?"(No doubt there will be some rude people who will tell me that I'm the last person who should be appealing to that principle.) I don't see that it's up to me to identify the flaw in an argument whose conclusion obviously does not follow from its premises, but it will certainly strengthen my case if I can point to some feature or features of the argument that are viable candidates for the office "flaw(s) in the argument." (If I couldn't do that much, if no one could, if no one, after much effort by very able people, was able to make any halfway plausible suggestion as to what one of the alleged flaws might be-that would be a good reason to re-open the question whether Parsons had presented a convincing example of the indeterminacy of identity.) After all, as I've always insisted, if the idea of "the burden of proof" (the burden of being the only one in the local community of discourse who is required to prove things) makes any sense outside the law ${ }^{15}$, here's the sense it makes: The burden of proof is borne by whoever it is that is trying to prove something. And Evans was the one who was trying to prove something, to wit, that indeterminate identity was impossible. The critics of Evans's reasoning were not trying to prove that indeterminate identity was possible or to

\footnotetext{
${ }^{15}$ In a criminal trial, for reasons that have nothing to do with dialectics and have everything to do with the necessity of constraining the power of the state, the burden of proof falls upon the state (or the Crown or the prosecution) and not upon the accused or the defense - the "burden," that is, of having to prove its assertions.
} 
prove anything else-or nothing beyond, "Evans's argument does not demonstrate the impossibility of indeterminate identity." The critics of Evans's reasoning were not even trying to prove that that reasoning was invalid - but only that one didn't have to regard it as valid.

My own candidate for "flaw in the argument" was the following (adapted to the informal presentation of the argument I presented a moment ago). ${ }^{16}$ The reasoning included this assertion: "Rubbers obviously does not have the property of being indeterminately identical with Rubbers." ${ }^{17}$ Is it "obvious" that Rubbers lacks the property of being indefinitely identical with Rubbers? Let's back away from this question for a moment, and ask a more general question: How should the friends of the possibility of indeterminate identity answer this question: Suppose that $x$ has the property $\mathrm{F}$ and that $y$ is indeterminately identical with $x$; can it be determinately true that $y$ lacks F? I say, as one who takes the idea of indeterminate identity seriously, that it seems entirely plausible to say that the answer to this question is No. I can't prove that the answer is No, but, then, in the present dialectical situation, it's not incumbent on me to prove that thesis or any other thesis; it's rather up to Evans, or to the proponents of Evans's argument, to prove that the answer is Yes. And he-or they-will want to prove that. For suppose the answer is No. Let $\mathrm{F}$ be the property of being indeterminately identical with Rubbers. We have supposed for the sake of argument that Trashers has that property. But then, if the answer to our question is No, Rubbers cannot determinately lack the property of being indeterminately identical with Rubbers - since it is indeterminately identical with something that has that property. And, therefore, the proponent of Evans's argument is not in a position to affirm the "obvious" premise of the argument that I mentioned a moment ago: that Rubbers lacks the property of being indefinitely identical with Rubbers. Obviously that premise is not

${ }^{16}$ Material Beings, loc. cit.

${ }^{17}$ In my informal presentation of Evans's reasoning, this statement isn't deduced from anything. It's just put forward as pretty obviously true. If I were being more faithful to Evans's text, I'd have got to this statement by deducing it from the perhaps even more evident statement 'It is not indeterminate whether Rubbers is identical with Rubbers'. In the discussion that follows in the text, I'll continue to represent Evans's argument in this way. 
determinately false- but it is not determinately true, either, and thus cannot properly be asserted.

In my discussion of Evans's argument in Material Beings, I used the intuitive idea illustrated in this informal reply to Evans to construct a formal semantics for a simple little language (a very simple language indeed, but Evans's argument could be formulated in it). The philosophical lessons of the semantics were perhaps not negligible, but they were not as important as the intuitive idea behind it: if $x$ has the property $\mathrm{F}$, and if $y$ is indeterminately identical with $x$, then it cannot be determinately true that $y$ lacks F. ${ }^{18}$

I'll close by remarking that this formal semantics has attracted some very strange - so it seems to me at any rate-commentary. Here is a typical example (from an article by Nicholas Smith) of the kind of commentary I have in mind.

Van Inwagen seems to be presenting a standard sort of set-theoretic model, and indeed makes free use of the relation of identity with which any ordinary set comes pre-equipped (both in specifying that pairs be genuinely twomembered, and when he says "If $x$ and $y, x \neq y$, are members of a pair ...")—but this is then in tension with the later claim that "The objects with which an object is paired are to be thought of as the objects such that it is indefinite whether that object is identical with them." We have been explicitly told that in a pairing $\{x, y\}, x$ and $y$ must be non-identical. Now we are told that $x$ and $y$ are to be thought of as indefinitely identical. I cannot make sense of this. If we are working with a standard set-theoretic model, then $x$ and $y$ are simply non-identical; if we are not, then unless we are given some other way to understand the presentation, we do not understand it at all. The ordinary

${ }^{18}$ I will mention two features of the semantics that were not exhibited in my statement of the "basic idea" because I think they're of some interest. First, although the semantics refuses to confer definite truth on 'It is not the case that it is indeterminate whether Rubbers has the property of being identical with Rubbers', it insists on conferring definite truth on 'It is not the case that it is indeterminate whether Rubbers is identical with Rubbers'. (See the previous note.) And, secondly, it insists on conferring definite truth on 'It is not the case that it is indeterminate whether Rubbers has the property of being identical with itself'. (The property of being a thing that is identical with Rubbers is not the same property as the property of being a thing that is identical with itself. For one thing their extensions are rather different. Graduate students are always telling me that these two properties somehow become identical "when Rubbers has them." If you understand that, I hope you'll explain it to me, because I don't.) 
understanding of set-theoretic models rules out van Inwagen's proposed interpretation of his construction-yet no other way of understanding the construction is presented. ${ }^{19}$

I will remark that this criticism ignores long passages in the work Smith is discussing that, I would guess, are devoted to the very points he raisesalthough I can't be sure because I'm not sure what those points are. But let that pass-for it has more to do with the boring (to you anyway) and entirely non-philosophical question whether Smith was fair to me than it has to do with any philosophical problems about indeterminate identity.

Let us turn to the philosophical points. What is the charge that Smith is bringing against me? I don't know because I don't know what is intended by the phrase 'van Inwagen's proposed interpretation of his construction'. The "construction" was supposed to divide the inferences expressible in a certain simple formal language into two classes, classes I labeled "valid" and "invalid" (it of course consigns some of the inferences comprised in Evans's argument to the class I called "invalid"). It certainly does that, and insofar as that is its purpose, it doesn't need an interpretation. What else did I do or say that the charge might be directed against? Well, having laid out the semantics, I presented some philosophical arguments intended to show that the friends of indeterminate identity should find the division the semantics produces at least plausible-I mean that they should find it plausible to suppose that the arguments the semantics classifies as valid and invalid have just those properties. (The arguments were of the same sort as the "Trashers"-"Rubbers" argument above.) But Smith does not mention these arguments.

In the end, I think I have to say that Smith's criticism of the semantics is no more than a reaction to certain heuristic idioms I used-the most important of which is the phrase 'are to be thought of'. (The core of his argument seems to be these three sentences: "We have been explicitly told that in a pairing $\{x, y\}, x$ and $y$ must be non-identical. Now we are told that $x$ and $y$ are to be thought of as indefinitely identical. I cannot make sense of this.") If I am right about this, Smith's criticism is an artifact of his taking this phrase more seriously than I intended it to be taken,

${ }^{19}$ Nicholas J.J. Smith, "Why Sense Cannot be Made of Vague Identity," Nô̂s 42:I (2008), pp. I-I6. The quoted text is on p. 7 . 
of his placing more dialectical weight on it than it was designed to bear. And why did I use this phrase, this phrase that was not designed to bear much dialectical weight? Well, it's quite common for philosophers who are trying to convey the intuitive motivation for a formal semantics to use the phrase 'are to be thought of'-knowingly to use it-in ways that, on analysis, can be seen to make no sense.

The most obvious example is provided by its use by writers on the semantics of quantified modal logic when they are trying to give their readers an intuitive grasp of what is "going on" in the model theory. Consider the following sentence: "The members of the universal domain that are not assigned to the actual world by a model are to be thought of as the things that, according to that model, do not actually exist but exist in other possible worlds"-a sentence I have made up but which is typical of things that are said in textbooks of modal logic. ${ }^{20}$ In my view, the idea of things that do not actually exist (whether they exist in other possible worlds or not) is nonsense. And, therefore, the sentence I have imagined is nonsense, for an invitation to "think of" certain of the objects contained in a model "as" so-and-sos is nonsense if the definition that has been provided for 'so-and-sos' is nonsense. But if sentences like my imaginary sentence are nonsense, they're very useful nonsense: like it or not, it's an empirical fact that nonsense of that kind helps students of the semantics of quantified modal logic to keep their bearings while they are picking their way through the complexities of the model theory.

I think that the critics of my semantics who have said something along the lines I've been discussing-Smith is not the only one-must think that the purpose of the semantics is somehow to explain the idea of indeterminate identity or to make it intelligible to an audience of its

${ }^{20}$ Here's a real and rather famous example of this sort of talk, although it does not contain the words 'are to be thought of'. In "Semantical Considerations on Modal Logic" [printed in Leonard Linsky (ed.) Reference and Modality (Oxford and London: Oxford University Press: 1971), pp. 63-72], Saul Kripke wrote (p. 65): "Intuitively, $\psi(\mathrm{H})$ [the domain of the world $\mathrm{H}]$ is the set of all individuals existing in $\mathrm{H}$. Notice that $\psi(\mathrm{H})$ need not be the same set for different arguments $H$, just as, intuitively, in worlds other than the real one, some actually existing individuals may be absent, while new individuals, like Pegasus, may appear." I think it is obvious that Kripke might just as well have written ' $\psi(\mathrm{H})$ is to be thought of as the set of individuals existing in $\mathrm{H}$ ' as 'Intuitively, $\psi(\mathrm{H})$ is the set of all individuals existing in $\mathrm{H}^{\prime}$. 
cultured despisers or to show that it's a coherent idea. And they suppose that a certain bit of heuristic whistle-talk ('The objects with which an object is paired are to be thought of as the objects such that it is indefinite whether that object is identical with them') — a mere aside, a throwaway line-was an essential part of that project.

Nothing could be further from the truth. Explaining what indeterminate identity is is not what the formal semantics is for. And it's not for making the idea of indeterminate identity intelligible, either, or for demonstrating that that idea is coherent. ${ }^{21}$ Those things are what the piles of trash and the Cabinet are for: Consider those cases (we, the friends of indeterminate identity, whisper seductively to the cultured despisers), and you'll gain some degree of understanding of the idea of indeterminate identity — and you'll see that it's a coherent idea, since you'll see that there are possible cases of it. The semantics is simply a device for dividing the arguments expressible in a certain formal language into two exhaustive and exclusive classes. The task of convincing students of the semantics that one of those classes is "the valid ones" and that the other is "the invalid ones" falls to the informal philosophical commentary on the semantics. And, if that task has been accomplished, we friends of indeterminate identity can point out to the cultured despisers that Evans's argument is to be found among the invalid ones. ${ }^{22}$

${ }^{21}$ At one place (p. 6), Smith quotes a passage in which I define certain terms used in the model theory and describes it as "van Inwagen's attempt to make sense of vague identity." At many places he refers to attempts (mine supposedly among them) to "model vague identity" or to "model vague identity within set theory." These two phrases call for comment, since it's not clear what they mean. It's true that I gave a model-theoretic definition (a definition couched in terms of ordinary set theory) of the predicate 'valid' as applied to the arguments expressible in a language that includes an "indeterminacy" operator and the identity sign. Does that mean that I attempted to "model vague identity"? Owing to the vagueness of that phrase, the question has no answer. But if my definition of validity was an attempt to "model vague identity," my attempt to model vague identity was not an attempt to explain or make sense of vague identity.

${ }_{22}$ This paper was presented and discussed at a workshop called "Metaphysical Indeterminacy: the state of the art" at the University of Leeds in May of 2009 and was composed for that occasion. I thank the other speakers at and participants in the conference for many helpful comments. I am particularly grateful to Elizabeth Barnes, Ross Cameron, Katherine Hawley, Daniel Nolan, and Robert Williams. 